\title{
Pulse shortening in a Nd:glass laser by gain reshaping and soliton formation
}

\author{
D. Kopf, F. X. Kärtner, K. J. Weingarten, and U. Keller \\ Ultrafast Laser Physics, Institute of Quantum Electronics, Swiss Federal Institute of Technology, \\ ETH Hönggerberg HPT, CH-8093 Zürich, Switzerland \\ Received July 25, 1994
}

\begin{abstract}
We demonstrate that intracavity filtering and soliton formation in actively mode-locked lasers can lead to pulse shortening by as much as a factor of 30 . Pulses as short as 310 fs have been generated from a Nd:glass laser that is mode locked only by an acousto-optic modulator.
\end{abstract}

Active mode locking (AML) of Nd:glass laser usually results in pulse trains with pulse lengths of 5 to $10 \mathrm{ps}^{1-3}$ We have generated pulses as short as $310 \mathrm{fs}$ from regeneratively actively mode-locked Nd:phosphate and silicate lasers. Two techniques are employed, net gain reshaping by a knife-edge, which acts as an intracavity filter, and soliton formation resulting from negative group-velocity dispersion (GVD) and self-phase modulation (SPM) in the cavity. These AML results help to clarify the recently observed passive mode-locking behavior of $\mathrm{Nd}$ :glass lasers. ${ }^{4,5}$

The cavity design is equivalent to the setup used in Ref. 4, except that the antiresonant Fabry-Perot saturable absorber is replaced by an acousto-optic modulator (AOM) (Fig. 1). The drive signal for the $\mathrm{AOM}$ is generated from the pulse train itself, which is referred to as regenerative AML. ${ }^{6}$ However, here we use the term AML for simplicity.

The effect of net gain reshaping, i.e., gain minus the frequency-dependent loss that is due to intracavity filtering, is shown in Fig. 2. The measured fluorescence curve (solid curve) of a quasi-homogeneously broadened Nd:phosphate glass is proportional to the saturated gain. Inserting the knife-edge (Fig. 1) introduces a tunable long-pass wavelength filter, ${ }^{4}$ which flattens the net gain profile over a bandwidth of approximately $10 \mathrm{~nm}$ (Fig. 2).

The Kuizenga-Siegman formula for AML, ${ }^{7}$ neglecting SPM and GVD contributions, would predict a pulse width of

$$
\tau_{a}=\sqrt[4]{D_{g} / M_{s}}, \quad \tau_{a, \mathrm{FWHM}}=2 \sqrt{\ln 2} \times \tau_{a},
$$

where $D_{g}=g / \Omega_{g}{ }^{2}$ is the gain dispersion, $M_{s}=$ $M \omega_{m}{ }^{2} / 2$ is the modulator strength, $g$ is the saturated gain, $M$ is the modulation depth, and $\omega_{m}$ is the modulation frequency. For a Lorentzian-shaped gain profile, $\Omega_{g}$ is the HWHM linewidth. If the line shape is of an arbitrary form, $\Omega_{g}$ represents an effective linewidth that corresponds to a Lorentzian linewidth with the same peak curvature. After flattening out the gain, we can estimate that the equivalent linewidth $\Omega_{g}$ is increased by a factor of 5 to 10 . The AML pulse width would then be reduced from $10 \mathrm{ps}$ (Nd:phosphate with $\Omega_{g} \approx 2 \pi \times 4.0 \times 10^{-12} \mathrm{~s}^{-1}$,
$M=0.01$, and $g=0.01$ ) by a factor of 3 , as it scales with the square root of the linewidth [Eq. (1)].

However, we observe much shorter pulses with the Nd:phosphate laser. The autocorrelation and the spectrum showed a transform-limited sech-shaped pulse of only $310 \mathrm{fs}$ (Fig. 3) when the knife-edge was adjusted for optimum net gain reshaping. Without gain reshaping the $\mathrm{Nd}$ :phosphate laser generates only unstable picosecond pulses with strong wings [similar to trace (a) of Fig. 3b of Ref. 4].

Using an inhomogeneously broadened Nd:silicate glass as the gain medium in the same setup, we obtained 330-fs-long pulses, but this time the knife-edge was not necessary and did not help in the pulse shortening. For an ideal inhomogeneous gain medium the saturated gain would be flat over the inhomogeneous linewidth, assuming equally distributed spectroscopic parameters. We confirmed this by observing that the $\mathrm{cw}$ operation of the $\mathrm{Nd}$ :silicate laser consisted of many longitudinal modes spread over a range of 8 to $10 \mathrm{~nm}$. The mode-locking mechanism then merely has to lock the already running frequencies together.

It is obvious that the AOM is too weak to shape femtosecond pulses [Eq. (1)]. Haus and Silberberg ${ }^{8}$ investigated the effects of GVD and SPM on active and passive mode locking. They found that both processes together may lead to pulse shortening by as much as a factor of 2.5 until instabilities arise. Here we give a simplified explanation of why, for the case of large enough negative GVD and SPM, pulse shortening can be much larger as a result of solitonlike pulse shaping. A detailed analysis that employs soliton perturbation theory will appear elsewhere. ${ }^{9}$

We model the actively mode-locked laser with GVD and SPM by the master equation ${ }^{8}$

$$
\begin{aligned}
T_{R} \frac{\partial}{\partial T} A(T, t) & =\left[-i D \frac{\partial^{2}}{\partial t^{2}}+i \delta|A(T, t)|^{2}\right] A(T, t) \\
+ & {\left[g-l+D_{g} \frac{\partial^{2}}{\partial t^{2}}-q(t)\right] A(T, t), }
\end{aligned}
$$

where $A(T, t)$ is the slowly varying field envelope, $T_{R}$ is the cavity round-trip time, $D$ is the amount of intracavity GVD, $\delta$ is the nonlinear coefficient that is due to SPM, $l$ is the round-trip loss, $q(t)=M[1-$ 


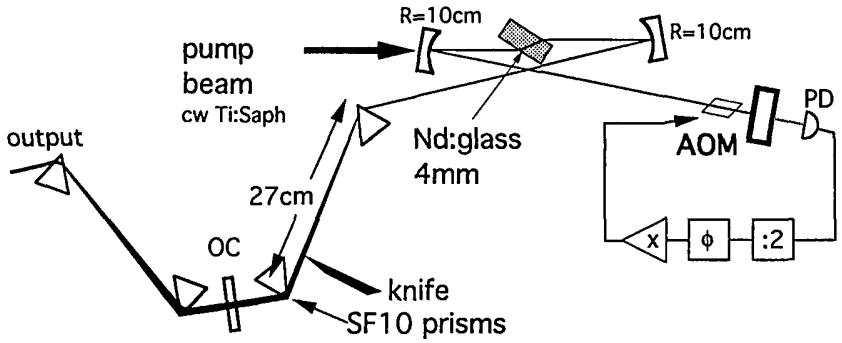

Fig. 1. Cavity design, a standard dispersion-compensated delta cavity with a mode radius of $35 \mu \mathrm{m} \times 55 \mu \mathrm{m}$ inside the gain medium. PD, photodiode.

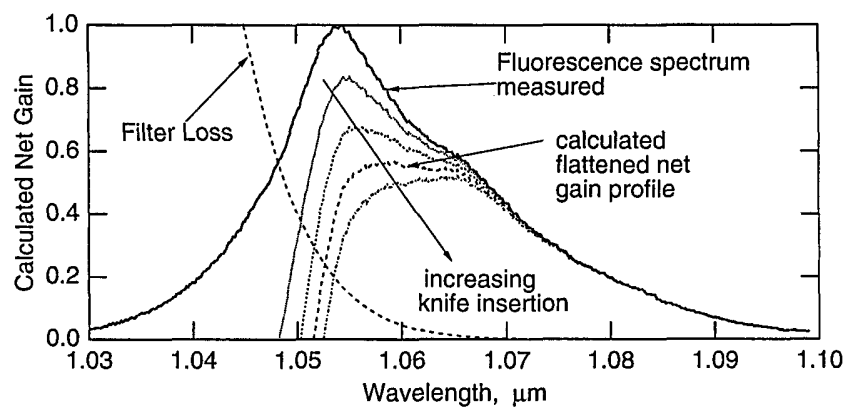

Fig. 2. Gain reshaping by insertion of a knife-edge into the laser beam between the two prisms (Fig. 1).

$\left.\cos \left(\omega_{m} t\right)\right]$ is the loss modulation that is due to the AOM, $g$ is the saturated gain $g=g_{0} /\left(1+W / E_{L}\right)$, $g_{0}$ is the unsaturated small-signal gain, $E_{L}$ is the saturation energy of the gain medium, and $W$ is the pulse energy.

The first part of Eq. (2) is the nonlinear Schrödinger equation, which has the well-known fundamental soliton solution $A_{s}(T, t)=\sqrt{W / 2 \tau} \operatorname{sech}(t / \tau)$ $\exp \left(i \Phi_{0} T / T_{R}\right)$, with $\Phi_{0}=\delta W / 4 \tau=|D| / \tau^{2}$, where the FWHM pulse width is given by $\tau_{\mathrm{FWHM}}=1.76 \tau$ and $\Phi_{0}$ is the phase shift of the soliton per round trip in the cavity. For the subpicosecond pulses that we have observed in our AML experiment the first two terms in Eq. (2) that are due to GVD and SPM are the dominant effects, which affect the pulse parameters pulse width and/or chirp on the order of $1 \%$ per round trip. The remaining terms, i.e., the effect of the net gain profile $D_{g} \partial^{2} / \partial t^{2}$ and the AOM modulation $q(t)$, are of the order of $0.01 \%$ (the AOM was operated off resonance). Assuming the soliton solution, we can derive the energy balance from Eq. (2), similar to the soliton storage ring ${ }^{10}$ :

$$
g-l=D_{g} /\left(3 \tau^{2}\right)+M_{s} \tau^{2} \pi^{2} / 12 .
$$

The soliton will saturate the gain until the steadystate condition is reached, i.e., gain is equal to the total losses per round trip.

In contrast to Ref. 10, where the authors were concerned with the growth of the noise in the time interval where no soliton is present, here we are concerned with the growth of instabilities in the presence of a soliton. Fortunately, one can show by soliton perturbation theory ${ }^{9}$ that these two problems are similar. This is not obvious, because it means that the coupling of the soliton to the continuum, where instabilities might grow, can be neglected. The dynamics of the instabilities then can be studied by Eq. (2) without SPM and with the gain saturated by the soliton. This result is intuitive because the AOM and the gain dispersion act as small perturbations on the soliton that generate a continuum too weak to experience SPM. Thus the stability requirement is that those instabilities decay with time.

In analogy to AML theory, ${ }^{11}$ the eigensolution of Eq. (2) without SPM that sees the most round-trip gain is given by a complex Gaussian pulse ${ }^{10} a(t)=$ $\exp \left(-\Gamma t^{2}\right)$, where $\Gamma=a-i b$ is a complex number. The FWHM pulse width of this Gaussian pulse is then given by $\tau_{\mathrm{FWHM}}^{2}=2 \ln 2 / a$. The complex Gaussian is introduced in Eq. (2) without SPM, and this gives us two equations for the case that the pulse energy and the pulse shape are conserved per round trip:

$$
\begin{aligned}
g_{I}-l & =2 D_{g} a-2 b D, \\
\Gamma & =\frac{1}{2 \tau_{a}^{2} \sqrt{1+i D_{n}}},
\end{aligned}
$$

where we have used Eq. (1) and $D_{n}=|D| / D_{g}$ as the normalized dispersion. Equation (4) describes the steady-state conditions for the Gaussian instability pulses, where $g_{I}$ is the round-trip gain required to compensate for the losses. As the gain medium is already saturated by the soliton in the cavity, the instabilities experience the same saturated gain $g$ of Eq. (3). This Gaussian instability mode vanishes in time when

$$
g<g_{I} \text {. }
$$

Combining Eqs. (3)-(5) and inequality (6) gives us a relation for the minimum amount of negative GVD for stable solitonlike pulse formation, $\tau_{a}^{2} / \tau^{2}+\tau^{2} \pi^{2} /\left(4 \tau_{a}^{2}\right)=3 \operatorname{Re} \sqrt{1+i D_{n}}$, which is in agreement with Ref. 9. In the asymptotic limit where $D_{n} \gg 1$, this turns into

$$
D_{n, \min }=\frac{2}{9} \times \sqrt[3]{\frac{\left(9 \phi_{0} / 2\right)^{2}}{M_{s} D_{g}}} .
$$

This minimum amount of negative GVD is necessary to spread the instabilities in time so they experience more loss from the AOM than the soliton experiences
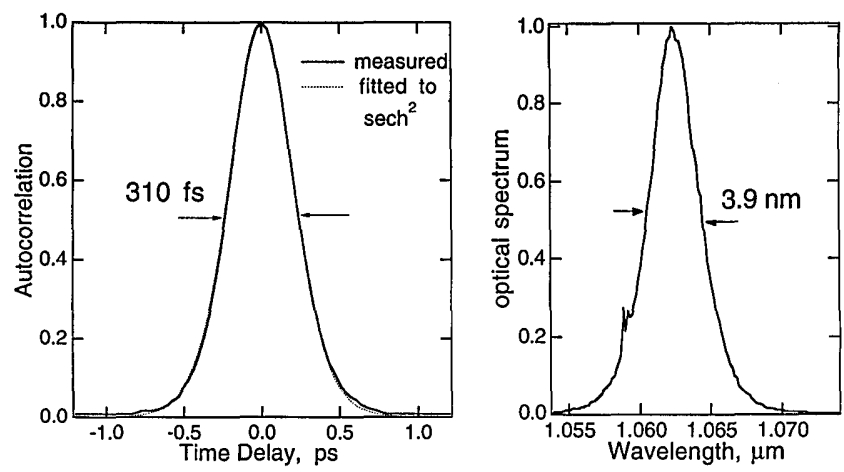

Fig. 3. Noncollinear measured autocorrelation trace fitted to an ideal $\operatorname{sech}^{2}$ pulse. The time-bandwidth product is 0.32 . Absorbed power $930 \mathrm{~mW}$, output power $70 \mathrm{~mW}$ at $1 \%$ output coupling. Total cavity losses are $2 \%$. 


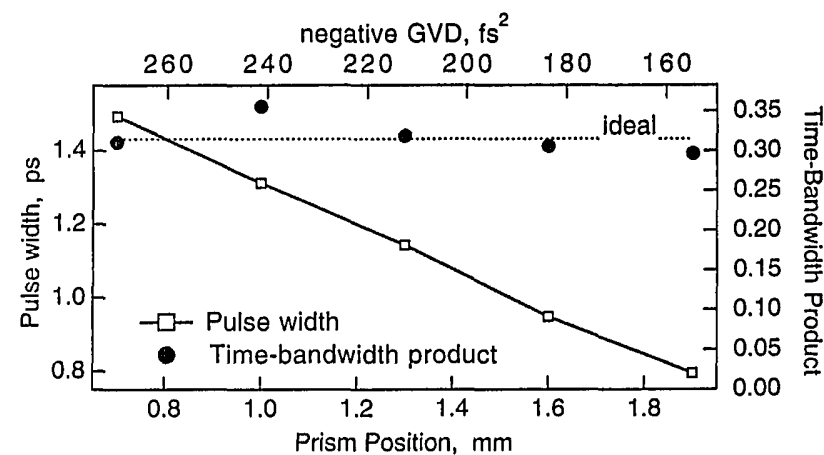

Fig. 4. Linear dependence of pulse width on intracavity negative GVD as expected for a soliton.

from gain filtering. The stability criterion is valid in particular for the Gaussian pulse that is the lowestorder eigenstate of Eq. (2) without the SPM term. Comparing the pulse width that we would expect in a pure AML system [Eq. (1)] with that which is due to soliton formation if sufficient GVD and SPM is present, we find for the pulse width reduction factor (using FWHM widths) that

$$
R=\frac{1.66}{1.76} \sqrt[4]{\frac{D_{g}}{M_{s}}} / \sqrt{\frac{D}{\phi_{0}}} \leq \frac{1.66}{1.76} \sqrt[12]{\frac{\left(9 \phi_{0} / 2\right)^{2}}{D_{g} M_{s}}} .
$$

As stability is guaranteed only as long as $D_{n}>D_{n, \min }$, as described in Eq. (7), we obtain a maximum reduction factor $R_{\max }$ for $D_{n}=D_{n, \min }$ [inequality (8)].

This is in good agreement with the experimental results, assuming 5 to 10 times net gain flattening, which means that $D_{g}$ is reduced by a factor of $10^{2}$, resulting in $R_{\max } \approx 10$. Therefore the FWHM pulse width in the AML case ( 3 ps in Nd:glass if gain reshaping is applied) is reduced by $R_{\max }$, resulting in $\approx 300$-fs-long pulses. Here we have used $\phi_{0}=0.01$, $M \approx 0.01, \omega_{m} \approx 2 \pi \times 240 \times 10^{6} \mathrm{~s}^{-1}, g \approx 0.01$, and $\Omega_{g}=10 \times 2 \pi \times 4.0 \mathrm{THz}$.

To show further that we are dealing with a soliton that is kept stable by the AOM and GVD against instabilities, we used a shorter crystal to reduce SPM and therefore to increase the pulse width to $\sim 1 \mathrm{ps}$. Then we tuned the intracavity negative GVD by moving one of the prisms and observed a linear dependence of the pulse width on GVD (Fig. 4), as expected for a soliton. The time-bandwidth product always stayed at 0.315 , confirming that we have a sech $^{2}$ shaped soliton rather than a Gaussian pulse, which we would expect in a pure AML experiment without SPM and where the time-bandwidth product would be at least 0.44 .

Kerr-lens mode locking (KLM) did not contribute to the experiment: The cavity was operated in the middle of the stability regime rather than close to its limits as is usually done with KLM lasers. In addition, mode locking occurred independently of the position of the gain medium. This we would not expect in a KLM laser, because the Kerr lensing effect can either enhance or suppress stable pulsing, depending on the gain medium position. Also, mode locking was never observed to be self-sustaining after the rf voltage of the AOM was turned off. Hard-aperture
$\mathrm{KLM}$ was shown not to be present in the Nd:silicate experiment, for which no gain reshaping has to be applied and therefore no hard aperture was used.

In conclusion, we have shown that soliton formation can lead to considerable pulse shortening, even in an AML laser, beyond the limits found numerically. ${ }^{8}$ The mechanism is summarized as follows. For the soliton, SPM and GVD are in balance, whereas the continuum where instabilities can grow experiences only GVD. Therefore, in contrast to the soliton, the continuum is spread in time so that the AOM can absorb it, which keeps the soliton stable. To reduce the influence of gain filtering on the soliton in the Nd:phosphate laser an intracavity filter had to be applied to flatten the net gain profile. With an inhomogeneously broadened Nd:silicate laser no intracavity filter was required, because spectral hole burning effectively flattened the gain profile. In the passively mode-locked Nd:glass laser demonstrated in Ref. 4 the same considerations now explain the buildup of femtosecond pulses after the insertion of a knife-edge acting as an intracavity filter. In that case the saturable absorber (i.e., an antiresonant Fabry-Perot saturable absorber) instead of the AOM stabilizes the soliton. As a direct extension of this result, we can show that a solitonlike pulse can also be stabilized by a slow saturable absorber alone, ${ }^{12}$ i.e., the relaxation time of the absorber can be much longer, typically by a factor of 20 , than the width of the soliton. This implies that semiconductor saturable absorbers with fast relaxation times near $100 \mathrm{fs}$ (thermalization time) can support pulses approaching the $10-\mathrm{fs}$ regime.

The authors thank Lightwave Electronics for the use of the AOM. This research was supported by the Swiss National Fund, grant 21-39362.93.

\section{References}

1. L. Yan, J. D. Ling, P.-T. Ho, and C. H. Lee, Opt. Lett. 11, 502 (1986).

2. S. Basu and R. L. Byer, Opt. Lett. 13, 458 (1988).

3. D. W. Hughes, M. W. Phillips, J. R. M. Barr, and D. C. Hanna, IEEE J. Quantum Electron. 28, 1010 (1992).

4. U. Keller, T. H. Chiu, and J. F. Ferguson, Opt. Lett. 18, 1077 (1993).

5. F. X. Kärtner, D. Kopf, and U. Keller, in Ultrafast Phenomena, Vol. 7 of 1994 OSA Technical Digest Series (Optical Society of America, Washington, D.C., 1994), paper MA5.

6. J. D. Kafka, M. L. Watts, and J.-W. J. Pieterse, IEEE J. Quantum Electron. 28, 2151 (1992).

7. D. J. Kuizenga and A. E. Siegman, IEEE J. Quantum Electron. QE-6, 694 (1970).

8. H. A. Haus and Y. Silberberg, IEEE J. Quantum Electron. QE-22, 325 (1986).

9. F. X. Kärtner, D. Kopf, and U. Keller, "Solitary pulse stabilization and shortening in actively mode-locked lasers," J. Opt. Soc. Am. B (to be published).

10. H. A. Haus and A. Mecozzi, Opt. Lett. 17, 1500 (1992).

11. H. A. Haus, IEEE J. Quantum Electron. 11, 323 (1975)

12. F. X. Kärtner and U. Keller, "Stabilization of solitonlike pulses with a slow saturable absorber," Opt. Lett. (to be published). 